\title{
Current issues in child and adolescent psychopharmacology. Part 1: Attention- deficit hyperactivity and affective disorders
}

\author{
Dave Coghill
}

\begin{abstract}
Drug treatments play an important role in the treatment of child and adolescent psychiatric disorders. However, there is often a long delay before research findings are translated into clinical practice; furthermore, changes in clinical practice outstrip the available evidence. This paper focuses on current issues and research findings on the pharmacological treatment of attention-deficit hyperactivity disorder (ADHD) and affective disorders. Clinical findings from a US study of the treatment of ADHD with extended-release stimulants and non-stimulants, and the development and use of clinical guidelines are discussed. Clinical trials of selective serotonin reuptake inhibitors for early-onset depression, approaches to managing treatment-resistant depression and guidance on the drug treatment of early-onset mania are considered.
\end{abstract}

It has become increasingly difficult to keep up with the rapidly developing evidence base in child and adolescent psychopharmacology over the past few years. A search of the Medline database over the past 5 years yields over 1000 published articles, indicating that the publication rate has almost doubled compared with the previous 15 years. Papers, posters and symposia on drug treatments increasingly dominate the programmes for international conferences on child and adolescent psychiatry, particularly those held in North America. In addition, members of online discussion groups frequently debate issues relating to the use of psychoactive medication for the treatment of a wide range child psychiatric problems.

One reason for the boom in research activity is a recent change in legislation in the USA with the introduction of the Food and Drug Administration Modernization Act 1997. This legislation requests that all new drugs that 'may produce health benefits in a paediatric population should have paediatric clinical trials and that already licensed drugs that have potential benefits to children should also be considered for such trials'. This has led to a surge of interest from researchers and the pharmaceutical industry in both general paediatric pharmacology and paediatric psychopharmacology, resulting in a huge increase in the number of paediatric clinical trials and in many new medications being granted licences for use in a paediatric population in the USA. While there is, as yet, no sign of similar legislation being proposed for the UK, a recent article in the medical press (Ganley, 2001) suggested that it is important for European governments to look at this issue more closely and that doing so would lead to safer evidenced-based treatments being made available to young people with psychiatric problems.

One of the greatest obstacles to evidence-based clinical practice is the time taken to translate research findings into treatment packages that are effective and usable within a non-specialised out-patient setting. Although this is as true with respect to the use of psychoactive medication in children as it is in other areas of medicine, there are also several examples where changes in our clinical practice have outstripped the available evidence. This everincreasing interest must also be seen against the backdrop of continuing debate, sometimes heated and often polarised, about the appropriate place that medication should play in our day-to-day practice. This two-part paper will describe some of the recent advances in child and adolescent psychopharmacology. I have chosen to structure the discussion by disorder rather than drug class, as this best reflects the ways in which clinical decisions are made about individual patients. Part 1 focuses on attentiondeficit hyperactivity disorder (ADHD) and the affective disorders; part 2 will look at anxiety

Dave Coghill is senior lecturer in child and adolescent psychiatry in the Department of Psychiatry, University of Dundee (Ninewells Hospital and Medical School, Dundee DD1 9SR, UK. Tel. 01382 204004; e-mail: david.coghill@tpct.scot.nhs.uk). $\mathrm{He}$ is also an honorary consultant child and adolescent psychiatrist in the Tayside Department of Child and Family Psychiatry. His main interests and research are in the neuropsychopharmacology of attention-deficit hyperactivity disorder and the psychopharmacology of child and adolescent psychiatric disorders. He is currently chair of the Royal College of Psychiatrists' Faculty of Child and Adolescent Psychiatry Standing Group on Psychoactive Medication. He has received consultancy fees and travel costs by Janssen-Cilag and Eli Lilly. 
disorders, obsessive-compulsive disorders, pervasive developmental disorders, schizophrenia and Tourette syndrome (Coghill, 2003).

\section{Hyperkinetic disorder/attention- deficit hyperactivity disorder}

There has been more research into the use of medication for the treatment of ADHD than into any other area of child and adolescent psychopharmacology, and it is still the case that about half of all papers published concerned with psychoactive medication and children are on the treatment of ADHD. Although many questions remain unanswered, there have been several key advances in knowledge over the past few years. Much of the research activity continues to be directed towards clinical studies, that there has also been a noticeable increase in basic science research. These basic science studies have helped to clarify the relationship between the pharmacokinetics and pharmacodynamics of stimulant medications and the action mechanisms of stimulant drugs. Similar studies have been carried out in general adult psychiatry for many years and, although basic pharmacological and neuroscience research may not sit as comfortably with child psychiatrists, it is important to recognise both their clinical relevance and the benefits they can ultimately bring to our patients.

\section{Current controversies}

The use of stimulant medications for the treatment of ADHD remains controversial. Pressure groups opposed to the use of medication frequently cite arguments which suggest that the troubles faced by children labelled as having ADHD are, in fact, the result of stimulant medication and that the use of stimulants in children leads to increased levels of substance misuse. It is certainly true that some children may have suffered adverse events as a result of being overmedicated, but recent studies have demonstrated clear neuropsychological deficits in children with ADHD who have never been exposed to psychostimulants or other psychoactive medications (Coghill et al, 2001). Studies into the relationship between ADHD and substance misuse have shown that there are not only similarities but also important differences between methylphenidate and other drugs of misuse such as cocaine. For example, Volkow et al (1995) describe an elegant series of studies demonstrating that, although these two drugs have similar mechanisms of action, the very different pharmacokinetic and pharmacodynamic properties of low-dose oral methylphenidate are likely to limit its potential as a drug of misuse. Clinical studies also suggest that, rather than increase the risk for later substance misuse, stimulant treatment seems to have a protective effect (e.g. Biederman et al, 1999). For those wishing to read more about the basic science of stimulant medications and ADHD, much of this research is described in more detail by Solanto et al (2001).

\section{Recent findings from the MTA study}

The publication at the end of 1999 of the primary findings from the USA's National Insitute for Mental Health Collaborative Multisite Multimodal Treatment Study of Children with Attention Deficit/ Hyperactivity Disorder (the MTA study; MTA Cooperative Group, 1999a,b) and the ensuing commentaries on and criticisms of the study marked a milestone in child and adolescent psychiatry research. The MTA study's primary findings have been well reported and discussed, both professionally and in the media. Over the past year, several further papers have been published by the group, extending their original findings. Four of these publications are of particular relevance to this discussion: Conners et al (2001), Swanson et al (2001), Greenhill et al (2001) and Vitiello et al (2001).

\section{Outcomes of the MTA study}

Although it is now generally accepted that medication can play an important part in the management of ADHD, one of the most difficult decisions facing clinicians planning and delivering services for children and young people with ADHD continues to be whether to offer a mainly pharmacological treatment package or a more-expensive and time-consuming package of combined psychosocial and pharmacological treatments. This was one of the primary questions addressed by the MTA group and it resulted in one of the study's more controversial conclusions that, despite there being a pattern of non-significant superiority for the combined treatment over the medication-only treatment on most of the outcome measures, 'combined behavioural intervention and stimulant medication multi-modal treatment, the current gold standard for ADHD interventions - yielded no statistically significant greater benefits than medication management for core ADHD symptoms' (Conners et al, 2001). This primary analysis measured outcome across a wide range of domains utilising a total of 19 different outcome measures. An analysis using multiple outcome measures may be of benefit in revealing specific patterns of outcome from the different treatment approaches, but it is generally accepted that limiting a clinical trial to a single outcome measure is usually a more appropriate option (Box 1). 
Box 1 Advantages of choosing a single outcome measure in clinical trials

It increases the power of a study to detect significant effects

It makes the results of a study easier to interpret (multiple measures often give conflicting results)

It is more effective at capturing true treatment effects in situations where there are unpredictable variations in disease state:

- from day to day

- between observers

- between measures

- when the treatments themselves have different effects across different aspects of functioning

In an attempt to address this issue, which can be interpreted as a flaw in the design of the MTA study, Conners et al (2001) conducted a post hoc analysis of the study using a single composite measure of treatment outcome derived from the mean scores on several of the standardised parent and teacher measures. This composite included ratings of externalising and internalising symptoms and of social skills. The resulting analysis confirmed most of the MTA results but found that, although the effect size for combined treatment $v$. medication-only management was the same as that previously reported $(0.28$, in the low-to-moderate range), this difference was now statistically significant. They concluded that, 'with the overall measure used in this analysis, combined multi-modal therapy has a clinically meaningful and statistically significant advantage over monotherapies and community treatments'.

Swanson et al (2001) also reanalysed the MTA data, this time using a categorical outcome measure, based on summed parent and teacher ratings of core ADHD and oppositional-defiant disorder symptoms, which defined children as 'successfully' or 'unsuccessfully' treated. They reported differences in success rate between the four groups (combined: 68\%; medication only: $56 \%$; behavioural only: $34 \%$; community care: $25 \%$ ) and also demonstrated a small but statistically significant effect of combined treatment over the medication-alone treatment. Interestingly, there were also unexpected but clear differences in outcome, depending on which site children were treated. At three sites, the behavioural treatment was better than the community treatment and at three sites the reverse was true. Thus, substituting the MTA behavioural treatment package for community care (which included the use of stimulant medication in approximately $60 \%$ of cases) may result in a positive or negative impact, depending on local conditions and clinical practice.
These analyses clearly add colour and help to clarify some of the messages from the MTA study. It is, however, important to note that they were both post hoc analyses which involved altering the end point of the trial after it had been run and they should not be seen as undoing the results of the original analysis, based on the a priori hypotheses and decision to use multiple outcomes.

\section{What can be learnt from the MTA medication management protocol?}

Two further papers from the MTA study have given us insights into the rational prescribing of stimulant medication (Greenhill et al, 2001; Vitiello et al, 2001). These papers are particularly important when considering how aspects of the MTA medication protocol, which was clearly much more effective than the community treatments, could be incorporated into day-to-day clinical practice. Greenhill and colleagues analysed the MTA data set to investigate aspects of the intensive 28-day doubleblind, placebo-controlled methylphenidate titration used in the trial. Vitiello and colleagues examined the trajectory of methylphenidate dosage over time in the MTA children, in order to assess how well the titration was able to predict the doses required for effective long-term treatment. The key findings from both studies, which provide useful insights into starting children on stimulant medication, are summarised in Box 2. One thing to note when looking at these results is that they are almost certainly affected by the treatment algorithm used in the study, which aimed for maximal effect with 'no room for improvement' and allowed a dose decrease only for moderate-to-severe side effects. Thus, clinicians were not allowed to test whether improvement could be maintained on a lower dose.

\section{New stimulant preparations}

The short half-lives (methylphenidate about 3 hours, dexamphetamine about 10 hours) of stimulant medications and their short duration of action (about 4 hours) when given as immediate-release preparations, give rise to a number of problems (Box 3). Although no long-acting preparations of stimulant medication are currently available in the UK, they have been available in the USA for over 10 years. Unfortunately, these wax-matrix-based preparations have not proved to be as effective as the immediate release preparations and they have been used by clinicians much less than was expected. They have a delayed onset of action, result in lower peak plasma levels, and are typified by a tailing off in plasma concentration after peaking at 3 hours. The problems associated with these preparations may 


\section{Box 2 Key findings from the secondary analyses of the MTA study}

Greenhill et al (2001)

Both parents and teachers are able to detect medication effects on symptom reduction in children, using daily ratings

Parents were the best source for adverse-event ratings

Teachers have a tendency to attribute worsened behaviour to medication

Methylphenidate has significant effects on reducing ADHD symptoms at the weekends

Both parent and teacher ratings of symptoms significantly attenuate after baseline assessments, which may result in an overestimation of the impact of medication on ADHD symptoms in clinical settings

Smaller children $(<25 \mathrm{~kg}$ ) should have daily dose limited to $35 \mathrm{mg}$ of methylphenidate or less

Children $>25 \mathrm{~kg}$ are evenly distributed with respect to best-dose across all levels of dosing and should be titrated up to $60 \mathrm{mg} /$ day unless prohibited by adverse events.

Vitiello et al (2001)

Most $(88 \%)$ of the children who were started on methylphenidate at the end of titration remained on this drug throughout the study

Although the titration-determined dose was significantly correlated with the end-of-maintenance dose, only $17 \%$ of children remained on both the same medication and dosage throughout the study

The mean number of pharmacological changes per child was 2.8

Over half the children had at least one medication change in the first 3 months of maintenance

The mean doses of methylphenidate rose over the course of the study from $30.5 \mathrm{mg} / \mathrm{day}$ to $34.4 \mathrm{mg} / \mathrm{day}$

Children receiving the combined medication and behavioural package ended the study on lower doses of methylphenidate than those receiving the pharmacotherapy only

be partly explained by a series of studies carried out by Swanson et al (1999). They demonstrated that, when given three times a day, afternoon doses of methylphenidate result in an attenuated response compared with the same dose given in the morning. This has been interpreted as demonstrating the development of acute tolerance, which wears off by the next day, although there may be other explanations such as a diurnal variation in either symptoms or drug response.

These problems, allied with the changes in US legislation noted above, have led to intense activity within the pharmaceutical industry, resulting in the

\section{Box 3 Problems with immediate-release stimulants}

If administered in the traditional way, the drug levels and effects are at their lowest during the most unstructured times of the school day (e.g. lunchtime, break time and travelling home)

Compliance is often a problem, particularly with teenagers

Schools can be unreliable and opposed to administering medication or may have policies that prohibit effective administration

Many children and adolescents worry about ridicule by peers but are unable, during the school day, to maintain privacy over their medication

Some simply forget to take their medication unless reminded development of new modified-release stimulant preparations. There are no claims that treatment of the core symptoms of ADHD with these newer preparations is superior to treatment with their immediate-release counterparts, that they have been designed to be taken once a day, eliminating the need for multiple daily dosing for many patients. Four new long-acting psychostimulant preparations have been approved by the FDA for use in the USA and one of these (Concerta ${ }^{\circledR}$ ) has been licensed for use in the UK. It is likely that one or more of the other preparations licensed in the US will also become available in the UK in the not-too-distant future. Each preparation has its own particular qualities, mainly related to the length of clinical response (Table 1). The preparation available in the UK utilises a novel drug-delivery system designed to be given once a day by means of the caplet's osmotic pump, with a timed drug-delivery system producing an ascending-pattern plasma drug level through the day. This is intended to counterbalance the acute tolerance that is thought to develop during the afternoon.

A transdermal preparation of methylphenidate has also been developed as a once-a-day patch and, if successful, this may produce more sustained levels of methylphenidate with a greater consistency in therapeutic response, low misuse potential and enhanced compliance. Peer-reviewed papers on this delivery system have yet to appear. Lastly, it has now become clear that pharmacological effects of methylphenidate are almost completely due to the 
Table 1 New modified-release preparations licensed for the treatment of ADHD in the USA

\begin{tabular}{|c|c|c|c|c|c|}
\hline Medication & $\begin{array}{l}\text { Dose } \\
(m g)\end{array}$ & $\begin{array}{l}\text { Dose range } \\
(m g / \text { day })\end{array}$ & $\begin{array}{l}\text { Peak effect } \\
\text { (hours) }\end{array}$ & $\begin{array}{l}\text { Duration of } \\
\text { action (hours) }\end{array}$ & $\begin{array}{l}\text { Published evidence } \\
\text { of efficacy and safety }\end{array}$ \\
\hline \multicolumn{6}{|l|}{ Methylphenidates } \\
\hline $\begin{array}{l}\text { OROS- } \\
\text { methylphenidate } \\
\left(\text { Concerta }^{\circledR}\right)\end{array}$ & $18 ; 36 ; 54$ & $18-54$ & 8 & 12 & $\begin{array}{l}\text { Keating et al (2001); Pelham et } \\
\text { al (2001); Wolraich et al (2001) }\end{array}$ \\
\hline Metadate $\mathrm{CD}^{\circledR}$ & 20 & $20-60$ & 5 & 8 & Anonymous $(2001 b)$ \\
\hline Ritalin LA ${ }^{\circledR}$ & 20 & $20-60$ & 5 & 8 & None available \\
\hline $\begin{array}{l}\text { Amphetamines } \\
\text { Adderall } \mathrm{XR}^{\mathrm{TM}}\end{array}$ & $10 ; 20 ; 30$ & $10-40$ & $1-4$ & $10-12$ & $\begin{array}{l}\text { Grcevich (2001); Greenhill et } \\
\text { al (2002) }\end{array}$ \\
\hline
\end{tabular}

d-threo isomer (Kimko et al, 1999). This has led to the production and licensing in the USA of a dexmethylphenidate preparation that comprises only this active isomer.

\section{Non-stimulant treatments for ADHD}

In addition to the difficulties that arise from the need to take multiple doses during the day, it is estimated that up to $30 \%$ of children with ADHD either do not respond to or cannot tolerate stimulant medications. This has led to a search for alternative, non-stimulant treatments. There is a strong literature stretching back many years supporting the efficacy of the tricyclic antidepressants in the treatment of ADHD. The evidence for other non-stimulant treatments such as clonidine and bupropion is, however, limited to a few clinical trials with relatively small numbers. Despite this, these treatments are frequently mentioned in the published treatment protocols and algorithms. The literature on the safety, efficacy and use of these medications has been reviewed by Biederman \& Spencer (2000).

Several new and promising non-stimulant medications for the treatment of ADHD are currently undergoing clinical trials. Atomoxetine (a specific noradrenaline reuptake inhibitor that failed trials as an antidepressant) has been shown to be effective and safe in treating ADHD in both adults (Spencer et al, 1998) and children (Michelson et al, 2001; Spencer et al, 2001). It has also been shown to have a low misuse potential. Work has also been carried out exploring the effects of nicotinic drugs in the treatment of adult ADHD. A small open trial of nicotine patches (Conners et al, 1996; Levin et al, 1998) and an exploratory randomised controlled trial of ABT-418, a central nervous system nicotinic agonist, also delivered via a transdermal system (Wilens et al, 1999), reported positive results, and it seems likely that in the next few years there will be several non-stimulant options available for treating ADHD.

\section{Guidelines and protocols for managing ADHD}

Aspects of the MTA study and other recent research have already had a profound influence on the development of clinical practice guidelines for the management of ADHD in both the UK and the USA (National Institute for Clinical Excellence, 2000; Hill \& Taylor, 2001; Scottish Intercollegiate Guidelines Network, 2001; American Academy of Child and Adolescent Psychiatry, 2002). There are now more similarities than differences between guidance to clinicians on each side of the Atlantic, although the UK guidance is still likely to lead to fewer children being started on medication than in the USA. Although the evidence suggests that stimulant medication is almost certainly the most powerful treatment for core ADHD symptoms, as stated by Santosh \& Taylor (2000) in their excellent and comprehensive review of the use of stimulant medication, 'the most powerful therapy is not always the one to choose'.

\section{Affective disorders}

\section{Depression}

The effectiveness of psychotherapeutic interventions, particularly cognitive-behavioural therapy and interpersonal therapy, has been demonstrated in the treatment of many children and adolescents with depressive symptoms and mildto-moderate depressive episodes. However, owing to the limited availability of trained cognitive and interpersonal therapists and the fact that a psychotherapeutic approach is sometimes unsuitable or ineffective, pharmacological treatments often need to be considered.

A recent Cochrane review (Hazell et al, 2000) has confirmed previous findings that tricyclic antidepressants are unlikely to be of benefit in the treatment of depression in pre-pubertal children and that, although there is marginal evidence to support 
their use in the treatment of depression in adolescents, benefits are likely to be moderate, at best. In view of the relative ineffectiveness of the tricyclics and the serious adverse events associated with their use, particularly in overdose, there has been a rapid increase in the use of the selective serotonin reuptake inhibitors (SSRIs) in children and adolescents. This increased use has outstripped the evidence base. There are, to date, only two published randomised controlled trials of SSRIs: one with fluoxetine and the other with paroxetine (Emslie et al, 1997; Keller et al, 2001). Both of these studies reported a positive response, at least over the short term, and low levels of adverse events. Numerous open-label studies support the efficacy of various SSRIs and several industry-sponsored trials are currently underway. It seems likely that, as all of the SSRIs have similar mechanisms of action, they will all prove of use in the treatment of early-onset depression. Other atypical antidepressants that have some preliminary support for use in this population are venlafaxine, which has both serotonin and noradrenaline reuptake inhibitory properties; nefazadone, a potent $5-\mathrm{HT}_{2 \mathrm{a}}$ postsynaptic antagonist and moderate serotonin and noradrenaline reuptake inhibitor; and bupropion, an atypical antidepressant with an unclear mechanism of action that has partial inhibitory effects on noradrenaline and dopamine. It is too early to say whether or not these drugs will fulfil these early expectations. The recent literature on treatment of early-onset depression has been well reviewed by Martin et al (2000).

What is clear is that not all children and adolescents with depression respond to treatment with SSRIs. For example, in Emslie et al's (1997) study, complete symptomatic remission was uncommon across all arms of the study, and seen in only one-third of the patients in the fluoxetine arm. There was also a high relapse rate during the first year: of those who initially recovered, $39 \%$ had a relapse during the 1-year follow-up period. It is estimated that, overall, at least $40 \%$ of children and adolescents with depression will fail to respond to an initial trial with an SSRI. This rate of response failure is similar to the rates reported in cognitivebehavioural therapy studies. Although there is, as yet, no empirical evidence for managing treatmentresistant child and adolescent patients, some guidance can be offered (Box 4). The Children's Medication Algorithm Project has developed a treatment algorithm for early-onset depression which is a useful guide for clinicians (Hughes et al, 1999). A major study entitled the Treatment of SSRIResistant Depression in Adolescents (TORDIA), sponsored by the US National Institute of Mental Health, is utilising several of the strategies detailed in Box 4 in an attempt to find empirical answers

\section{Box 4 Management of treatment-resistant depression when an SSRI has failed}

Ensure adequate dose of the single treatment (e.g. paroxetine $20 \mathrm{mg}$ ) for sufficient duration (e.g. 4 weeks, followed by 4 weeks at the maximum tolerated dose

Re-evaluate:

(a) diagnosis/comorbidity, including sub stance misuse

(b) medical illnesses

(c) adherence to treatment

(d) exposure to negative events, e.g. abuse

(e) family functioning

Educate about treatment-resistant depression

Consider the treatment options:

(a) combined psychotherapy and medication

(b) switching to another treatment, e.g.

- within class, to another SSRI

- to another class (e.g. venlafaxine, nefazadone, bupropion)

(c) augmentation (no empirical data)

- add psychotherapy (interpersonal or cognitive-behavioural therapy)

- add an SSRI or bupropion

- add lithium or clomipramine (watch for 'serotonin syndrome')

to many of the questions in this area (http:// www.wpic.pitt.edu/research/Tordia).

Psychotherapeutic and pharmacological therapies seem to be roughly similar in potency for treating early-onset depression, but there are no head-to-head comparisons of these two approaches, nor is there any information about the relative effectiveness of a combined-treatment approach in contrast to either approach used alone. Large multisite studies are currently being carried out in the UK and USA in an attempt to answer these questions.

\section{Manic episodes and bipolar affective disorder}

There are now many published papers, mostly from the USA, on the pharmacological treatment of earlyonset bipolar disorder. Unfortunately, this literature is difficult to untangle. Many of the problems arise from disagreements on the most appropriate way to apply current diagnostic criteria, designed for use in adults, to children and adolescents. This has led to uncertainty over how best to define caseness and uncertainty about prevalence. Studies on adults with bipolar disorder estimate that between $20 \%$ and $54 \%$ report onset of symptoms during childhood (Lish 
Box 5 Deciding the drug treatment for earlyonset manic episodes and bipolar affective disorder (after Davanzo \& McCracken, 2000)

The following factors should be taken into account in deciding on the appropriate drug treatment:

Type of presentation

Euphoric: first line, lithium; second line, valproic acid

Mixed dysphoric: first line, valproic acid; second line, carbamazepine; third line, lithium

Psychotic: first line, valproic acid or lithium with an atypical or conventional antipsychotic

Rapid cycling: first line, valproic acid; second line, valproic acid and carbamazepine

Pattern of illness

A depression-mania inter-episode is more likely to respond to lithium than is a maniadepression inter-episode

Family history of mood disorders

A positive family history of bipolar disorder is correlated with lithium responsiveness

Gender

Care must be taken in treating adolescent girls with valproic acid, owing to the possible risk of polycystic ovaries

The side-effect profile of each patient

et al, 1994). Data from community samples suggest that the lifetime prevalence of bipolar disorder in adolescents is about $1 \%$, with an additional $5 \%$ reporting a distinct period of abnormally and persistently elevated, expansive or irritable mood, not meeting criteria for bipolar disorder but resulting in significant impairment and associated with high levels of comorbidity (Lewinsohn et al, (1995).

Manic episodes are notoriously difficult to diagnose with confidence in children and adolescents. The definitions regarding the severity of mania are unclear, leading to the blurring of diagnostic boundaries. The situation is further complicated by the fact that mania shares symptoms with other common disorders such as ADHD, depression and anxiety, including irritability, hyperactivity, poor judgement and reduced sleep.

In the UK, it is still rare to make a diagnosis of bipolar disorder, but the situation in the USA is very different. The DSM-IV diagnosis (American Psychiatric Association, 1994) of 'bipolar disorder not otherwise specified' is commonly used to diagnose adolescents presenting with exacerbations of disruptive behaviour, moodiness, low frustration tolerance and explosive anger, followed by guilt, hyperactivity, depression and difficulty sleeping at night. In children, this is often extended to chronic irritability and prolonged temper tantrums. Mood stabilisers are being used to target several of these symptoms for children who, in the UK, would be more likely to be given other diagnostic labels. It seems likely that the 'truth' is somewhere in the middle, but it is simply not yet clear exactly where the line should be drawn. Further discussion of this problem is beyond the scope of this article but is well reviewed by James \& Javaloyes (2001).

Therefore it is often difficult to know exactly which patients have been included in clinical trials for the treatment of early-onset bipolar disorder and how this should be translated into clinical practice. Notwithstanding these difficulties, it does seem to be the case that true manic episodes are difficult to modify without medication. Case series and openlabel trials have provided some evidence for the efficacy of lithium, valproic acid (divalproex in the USA) and carbamazepine in the treatment of earlyonset mania (Davanzo \& McCracken, 2000). On the basis of this evidence, these authors drew up a treatment algorithm and made several suggestions as to which drug should be considered for which patient (Box 5). Despite the rather flimsy nature of the evidence on which this guidance is based, it is helpful in deciding how to treat these difficult patients.

\section{Conclusions}

There has been an increasing acceptance that medication plays an important part in planning treatment for children and adolescents with ADHD, depression and bipolar affective disorder, and this has led to increases in the prescribing of psychoactive medications for these disorders. Never the less, there are still many unanswered questions. Several large studies currently underway will help to answer these in much the same way as the MTA study has increased our knowledge with respect to the treatment of ADHD. There are also several new preparations and medications on the horizon that have the potential to bring benefits to our patients.

\section{References}

American Academy of Child and Adolescent Psychiatry (2002) Practice parameter for the use of stimulant medications in the treatment of children, adolescents, and adults. Journal of the American Academy of Child and Adolescent Psychiatry, 41, 26S-49S.

American Psychiatric Association (1994) Diagnostic and Statistical Manual of Mental Disorders (4th edn) (DSM-IV). Washington, DC: APA

Anonymous (2001b) Another long-acting methylphenidate (Metadate CD). Medical Letter on Drugs and Therapeutics, $43,83-84$ 
Biederman, J. \& Spencer, T. (2000) Non-stimulant treatments for ADHD. European Child and Adolescent Psychiatry, 9 (suppl. 1), I51-I59.

-, Wilens, T., Mick, E., et al (1999) Pharmacotherapy of attention-deficit/hyperactivity disorder reduces risk for substance use disorder. Pediatrics, 104, e20.

Coghill, D. R. (2003) Current issues in child and adolescent psychopharmacology. Part 2: Anxiety and obsessivecompulsive disorders, autism, schizophrenia and Tourette syndrome. Advances in Psychiatric Treament, 9, in press.

-, Rhodes, S. M., Thrower, M., et al (2001) A neuropsychological investigation of psychostimulant drug-treatment naive boys with hyperkinetic disorder (ADHD). Journal of Psychopharmacology, 15, A55.

Conners, C. K., Levin, E. D., Sparrow, E., et al (1996) Nicotine and attention in adult attention-deficit hyperactivity disorder (ADHD). Psychopharmacology Bulletin, 32, 67-73.

_, Epstein, J. N., March, J. S., et al (2001) Multimodal treatment of ADHD in the MTA: an alternative outcome analysis. Journal of the American Academy of Child and Adolescent Psychiatry, 40, 159-167.

Davanzo, P. A. \& McCracken, J. T. (2000) Mood stabilizers in the treatment of juvenile bipolar disorder. Advances and controversies. Child and Adolescent Psychiatric Clinics of North America, 9, 159-182.

Emslie, G. J., Rush, A. J., Weinberg, W. A., et al (1997) A double-blind, randomized, placebo-controlled trial of fluoxetine in children and adolescents with depression. Archives of General Psychiatry, 54, 1031-1037.

Ganly, C. (2001) Want restrictions eased on use of new drug for psychiatric illness in young. Irish Medical Times, 30 November, p. 6

Grcevich, S. (2001) SLI381: a long-acting psychostimulant preparation for the treatment of attention-deficit hyperactivity disorder. Expert Opinion on Investigational Drugs, 10, 2003-2011.

Greenhill, L. L., Swanson, J. M., Vitiello, B., et al (2001) Impairment and deportment responses to different methylphenidate doses in children with ADHD: the MTA titration trial. Journal of the American Academy of Child and Adolescent Psychiatry, 40, 180-187.

-, Findling, R. L., Swanson, J. M., et al (2002) A doubleblind, placebo-controlled study of modified-release methylphenidate in children with attention-deficit/ hyperactivity disorder. Pediatrics, 109, E39.

Hazell, P., O'Connell, D., Heathcote, D., et al (2000) Tricyclic drugs for depression in children and adolescents. Cochrane Library, issue 4. Oxford: Oxford Update Software.

Hill, P. \& Taylor, E. (2001) An auditable protocol for treating attention-deficit/hyperactivity disorder. Archives of Disease in Childhood, 84, 404-409.

Hughes, C. W., Emslie, G. J., Crismon, M. L., et al (1999) The Texas Children's Medication Algorithm Project: report of the Texas Consensus Conference Panel on Medication Treatment of Childhood Major Depressive Disorder. Journal of the American Academy of Child and Adolescent Psychiatry, 38, 1442-1454.

James, A. C. \& Javaloyes, A. M. (2001) The treatment of bipolar disorder in children and adolescents. Journal of Child Psychology and Psychiatry and Allied Disciplines, 42, 439-449.

Keating, G. M., McClellan, K. \& Jarvis, B. (2001) Methylphenidate (OROS formulation). CNS Drugs, 15, 495-500.

Keller, M. B., Ryan, N. D., Strober, M., et al (2001) Efficacy of paroxetine in the treatment of adolescent major depression: a randomized, controlled trial. Journal of the American Academy of Child and Adolescent Psychiatry, 40, 762-772.

Kimko, H. C., Cross, J. T., \& Abernethy, D. R. (1999) Pharmacokinetics and clinical effectiveness of methylphenidate. Clinical Pharmacokinetics, 37, 457-470.

Levin, E. D., Conners, C. K., Silva, D., et al (1998) Transdermal nicotine effects on attention. Psychopharmacologia, 140, 135-141.

Lewinsohn, P. M., Klein, D. N. \& Seeley, J. R. (1995) Bipolar disorders in a community sample of older adolescents: prevalence, phenomenology, comorbidity and course.
Journal of the American Academy of Child and Adolescent Psychiatry, 34, 454-463.

Lish, J. D., Dime-Meenan, S., Whybrow, P. C., et al (1994) The National Depressive and Manic-Depressive Association (DMDA) survey of bipolar members. Journal of Affective Disorders, 31, 281-294.

Martin, A., Kaufman, J. \& Charney, D. (2000) Pharmacotherapy of early-onset depression. Update and new directions. Child and Adolescent Psychiatric Clinics of North America, 9, 135-157.

Michelson, D., Faries, D., Wernicke, J., et al (2001) Atomoxetine in the treatment of children and adolescents with attention-deficit/hyperactivity disorder: a randomized, placebo-controlled, dose-response study. Pediatrics, 108, e83.

MTA Cooperative Group (1999a) A 14-month randomized clinical trial of treatment strategies for attention-deficit/ hyperactivity disorder. The MTA Cooperative Group. Multimodal Treatment Study of Children with ADHD. Archives of General Psychiatry, 56, 1073-1086.

- $(1999 b)$ Moderators and mediators of treatment response for children with attention-deficit/hyperactivity disorder: the Multimodal Treatment Study of Children with attention-deficit/hyperactivity disorder. Archives of General Psychiatry, 56, 1088-1096.

National Institute for Clinical Excellence (2000) Guidance on the Use of Methylphenidate (Ritalin, Equasym) for Attention Deficit/Hyperactivity Disorder (ADHD) in Childhood, p. 13 London: NICE.

Pelham, W. E., Gnagy, E. M., Burrows-Maclean, L., et al (2001) Once-a-day Concerta methylphenidate versus three-times-daily methylphenidate in laboratory and natural settings. Pediatrics, 107, e105.

Santosh, P. J. \& Taylor, E. (2000) Stimulant drugs. European Child and Adolescent Psychiatry, 9 (suppl. 1), I27-I43.

Scottish Intercollegiate Guidelines Network (2001) Attention Deficit and Hyperkinetic Disorders in Children and Young People: A National Clinical Guideline, p. 52. Edinburgh: SIGN.

Solanto, M. V., Arnsten, A. F. \& Castellanos, F. X. (2001) Stimulant Drugs and ADHD: Basic and Clinical Neuroscience. New York: Oxford University Press.

Spencer, T., Biederman, J., Wilens, T., et al (1998) Effectiveness and tolerability of tomoxetine in adults with attentiondeficit hyperactivity disorder. American Journal of Psychiatry, 155, 693-695.

- - Heiligenstein, J., et al (2001) An open-label, doseranging study of atomoxetine in children with attentiondeficit hyperactivity disorder. Journal of Child and Adolescent Psychopharmacology, 11, 251-265.

Swanson, J., Gupta, S., Guinta, D., et al (1999) Acute tolerance to methylphenidate in the treatment of attentiondeficit hyperactivity disorder in children. Clinical Pharmacology and Therapeutics, 66, 295-305.

Swanson, J. M., Kraemer, H. C., Hinshaw, S. P., et al (2001) Clinical relevance of the primary findings of the MTA: success rates based on severity of ADHD and ODD symptoms at the end of treatment. Journal of the American Academy of Child and Adolescent Psychiatry, 40, 168-179.

Vitiello, B., Severe, J. B., Greenhill, L. L., et al (2001) Methylphenidate dosage for children with ADHD over time under controlled conditions: lessons from the MTA. Journal of the American Academy of Child and Adolescent Psychiatry, 40, 188-196.

Volkow, N. D., Ding, Y. S., Fowler, J. S., et al (1995) Is methylphenidate like cocaine? Studies on their pharmacokinetics and distribution in the human brain. Archives of General Psychiatry, 52, 456-463.

Wilens, T. E., Biederman, J., Spencer, T. J., et al (1999) A pilot controlled clinical trial of ABT-418, a cholinergic agonist, in the treatment of adults with attention-deficit hyperactivity disorder. American Journal of Psychiatry, 156, 1931-1937.

Wolraich, M. L., Greenhill, L. L., Pelham, W., et al (2001) Randomized, controlled trial of oros methylphenidate once a day in children with attention-deficit/hyperactivity disorder. Pediatrics, 108, 883-892. 


\section{Multiple choice questions}

1 Children weighing less than $25 \mathrm{~kg}$ should:

a not be started on methylphenidate

$\mathrm{b}$ have their dose limited to $35 \mathrm{mg} /$ day

c have methylphenidate titrated across the entire dose range

d not receive methylphenidate at weekends

e start on stimulant medication only after a full trial of psychosocial treatment.

2 When assessing response to methylphenidate:

a teachers are better than parents at rating adverse events

b parents should be the main informants for rating symptom improvement

c many children will require further changes in dose even after a successful titration

d adding effective psychosocial treatments may allow for lower dose of medication

e it is important to take baseline ratings.

3 As regards its pharmacological actions:

a atomoxetine is a non-specific noradrenaline reuptake inhibitor

b venlafaxine has both serotonin and noradrenaline reuptake inhibitory properties

c bupropion is a specific serotonin reuptake inhibitor

$\mathrm{d}$ nefazadone is a potent $5 \mathrm{HT}_{2 \mathrm{a}}$ postsynaptic antagonist

e paroxetine is an indirect dopamine agonist.
4 The potential benefits of using a single outcome measure in clinical trials include:

a increasing the power of the study

$b$ easier interpretation of results

c identifying specific patterns of outcome from different treatment approaches

d capturing true treatment effects in situations where there are unpredictable variations in disease state

e removing the need for a placebo arm.

5 Regarding early-onset bipolar affective disorder:

a lithium is the recommended first-line drug treatment for euphoria

b carbamazepine is the recommended first-line drug treatment for mixed dysphoria

c valproic acid is the recommended first-line drug treatment for rapid cycling

d a positive family history of bipolar affective disorder is correlated with poor lithium responsiveness

e data from RCTs support the effectiveness of lithium, carbamazepine and valproic acid in these cases.

\section{MCQ answers}

\begin{tabular}{|c|c|c|c|}
\hline 1 & 2 & 3 & 4 \\
\hline $\mathrm{F}$ & a F & a $\mathrm{F}$ & a $\mathrm{T}$ \\
\hline $\mathrm{T}$ & $b \quad F$ & b $\mathrm{T}$ & b $\mathrm{T}$ \\
\hline $\mathrm{F}$ & c $\mathrm{T}$ & c $\mathrm{F}$ & C F \\
\hline $\mathrm{F}$ & $\mathrm{d} T$ & $\mathrm{~d} T$ & $\mathrm{~d} T$ \\
\hline $\mathrm{F}$ & e $\mathrm{T}$ & e $F$ & e F \\
\hline
\end{tabular}

